\title{
Endobronchial Valve Placement as Destination Therapy for Recurrent Pneumothorax in the Setting of Advanced Malignancy
}

\author{
Christopher R Gilbert DO MSc, Jennifer W Toth MD, Umar Osman MD, and Michael F Reed MD
}

\begin{abstract}
The development of a persistent air leak after pneumothorax can be encountered in patients with underlying structural lung disease. In those with advanced malignancy or other comorbidities, the ability to tolerate general anesthesia and thoracoscopic procedures may limit definitive management. We describe the case of a 68-y-old male with refractory acute myelogenous leukemia presenting with recurrent secondary spontaneous pneumothorax and persistent air leak related to an underlying fungal pneumonia. Endobronchial valve placement allowed for timely chest tube removal and discharge from the hospital, as well as avoidance of a thoracoscopic procedure and pleurodesis. Key words: endobronchial valve; pneumothorax; persistent air leak; destination therapy. [Respir Care 2015;60(3):e46-e48. (C) 2015 Daedalus Enterprises]
\end{abstract}

\section{Introduction}

The development of a persistent air leak in patients with advanced malignancy can present a challenging therapeutic dilemma. Standard surgical management of recurrent pneumothorax and persistent air leak involves thoracoscopic procedures with the goal of pleurodesis and correction of the underlying cause. In patients with advanced malignancy or other significant comorbidities, less invasive therapeutic interventions may offer benefits in overall quality of life and use of health-care resources.

\section{Case Report}

A 68-y-old man with recurrent acute myelogenous leukemia and severe pancytopenia experienced acute dyspnea. Chest radiography revealed a large right pneumo-

Drs Gilbert, Toth, and Osman are affiliated with the Division of Pulmonary, Allergy, and Critical Care Medicine, and Dr Reed is affiliated with the Division of Thoracic Surgery, Milton S Hershey Medical Center, Penn State College of Medicine, Hershey, Pennsylvania.

Drs Toth and Reed are members of the clinical advisory board for Spiration. Drs Gilbert and Osman have disclosed no conflicts of interest.

Correspondence: Christopher R Gilbert DO MSc, Division of Pulmonary, Allergy, and Critical Care Medicine, Milton S Hershey Medical Center, Penn State College of Medicine, 500 University Drive, MCH041, Hershey, PA 17033. E-mail: cgilbert1@hmc.psu.edu.

DOI: $10.4187 /$ respcare. 03540 thorax (Fig. 1A). Repeat chest imaging after tube thoracostomy revealed adequate lung re-expansion, but a persistent air leak was observed.

Physical exam revealed subcutaneous emphysema within his neck and face. A continuous air leak on suction was identified. Computed tomography demonstrated proper chest tube placement along with multiple cavitary lesions and apical bleb disease (Fig. 1B). Previous bronchoalveolar lavage had demonstrated Aspergillus fumigatus, prompting ongoing antifungal therapy with voriconazole. Recent bone marrow aspiration demonstrated blast cells and recurrent acute myelogenous leukemia.

Multidisciplinary consultation with interventional pulmonology, oncology, and thoracic surgery considered treatment options for his persistent air leak in the setting of pancytopenia and refractory acute myelogenous leukemia. His overall poor prognosis and profound pancytopenia made operative management prohibitive not only secondary to resultant morbidity, but also variable success.

His air leak persisted, and on hospital day 5, bronchoscopy with balloon occlusion (B5-2C, Olympus, Center Valley, Pennsylvania) was performed. Balloon occlusion of the right upper lobe apical segment produced almost complete cessation of the air leak. One 7-mm and two 6-mm endobronchial valves (Spiration, Redmond, Washington) were placed into the right upper lobe apical segment, resulting in complete cessation of the air leak postoperatively. His chest tube was removed, and he was discharged home within $48 \mathrm{~h}$. His endobronchial valves were removed 8 weeks later. 

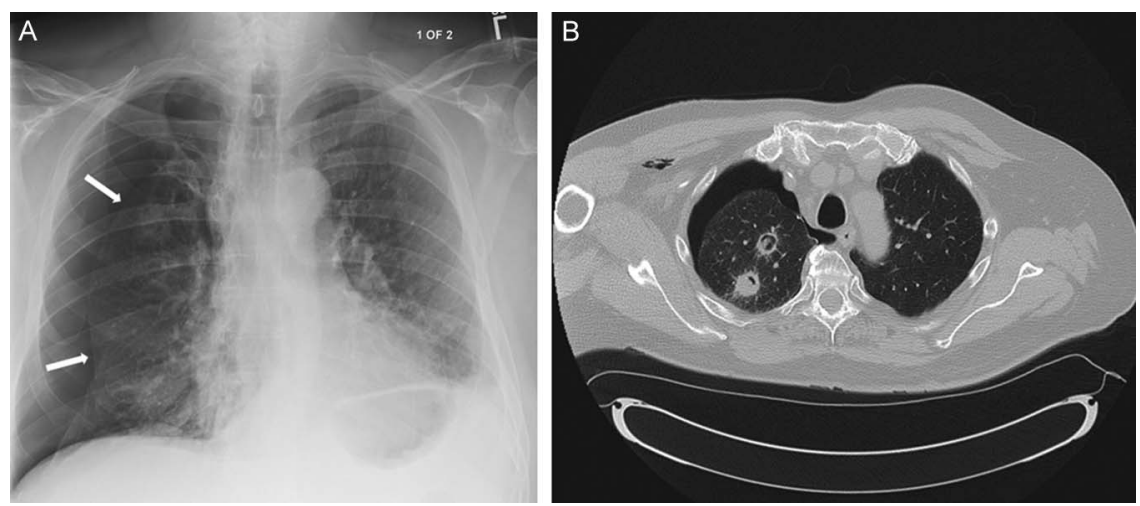

Fig. 1. A: Chest radiograph at initial presentation. The presence of a large right pneumothorax is identified (arrows). B: Axial image from the patient's chest computed tomography at presentation. Two cavitary lesions consistent with his Aspergillus pneumonia are present within the right lung parenchyma.

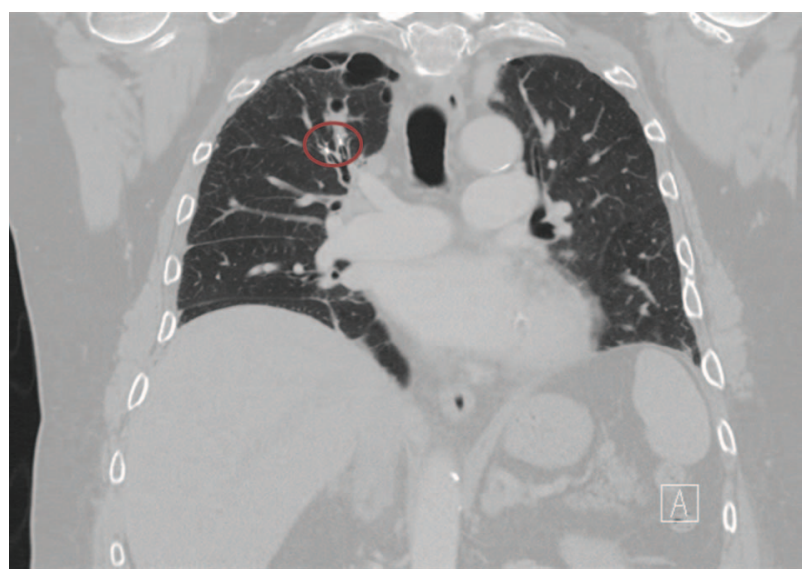

Fig. 2. Coronal image from the patient's chest computed tomography after placement of endobronchial valves. Two endobronchial valves are identified within the apical segment of the right upper lobe (red circle).

Approximately 3 weeks later, he experienced dyspnea, with chest imaging demonstrating a right pneumothorax. Tube thoracostomy was performed with subsequent lung re-expansion; however, the air leak again persisted. Due to his progressive hematologic malignancy and increasing need for transfusion support, the multidisciplinary team again recommended placement of endobronchial valves. However, at this time, it was advised that the endobronchial valves remain as a destination therapy in light of his end-stage malignancy and plans for future out-patient hospice care.

On hospital day 6, bronchoscopy with balloon occlusion and endobronchial valve placement was performed within the right upper lobe apical segment with cessation of the air leak. The patient's chest tube was removed, and he was discharged to home hospice within 48 h (Fig. 2). He required no further interventions. He died 7 months later from disease progression, experiencing no complications related to recurrent pneumothorax or endobronchial valve placement.

\section{Discussion}

Common treatment options for persistent air leaks involve continued tube thoracostomy drainage, pleurodesis, and surgical repair. ${ }^{1}$ Endobronchial valve placement remains the only endobronchial intervention available, with documented literature supporting use in air leaks. ${ }^{2-4}$ Although other interventions such as glue and coiling have been reported, the American College of Chest Physicians' statement on prolonged air leaks in pneumothorax suggest avoidance of these endobronchial interventions. ${ }^{5}$ Our multidisciplinary team pursued endobronchial valve placement in this patient with a persistent air leak after recurrent pneumothorax in the setting of an advanced refractory malignancy. Standard management options appeared unfavorable due to his underlying comorbidities; prolonged chest tube drainage via an out-patient Heimlich valve was undesirable due to persistent neutropenia and infectious complications, and thoracoscopic surgery in transfusiondependent acute myelogenous leukemia carries significant morbidity and mortality. Our strategy offered a minimally invasive method to resolve his air leak, permit early hospital discharge, and potentially decrease health-care cost for this patient with an incurable malignancy and limited life span. Although no clear guidelines exist for patient selection, in those with a known limited life span (and/or planned hospice referral), the avoidance of further invasive procedures should be strongly considered, which may deviate from manufacturer recommendation of endobronchial valve removal.

Endobronchial valves are currently FDA-approved as Humanitarian Use Devices in persistent air leaks after pulmonary resection, and a review of the indications and procedural technique has been recently published. ${ }^{4} \mathrm{Re}-$ 
ports of off-label endobronchial valve use continue to increase and have been documented in numerous situations at this point, including a heterogeneous case series of persistent air leaks. ${ }^{2}$ In patients in whom the leak can be isolated to an anatomic area (subsegmental, segmental, or lobar bronchi), endobronchial valve use provides a nonsurgical approach to the treatment of prolonged air leaks. In situations in which the leak cannot be identified, options such as prolonged intercostal tube drainage or pleurodesis may be required. This case appears to be the first reported use of endobronchial valves as planned destination therapy for recurrent pneumothorax in a patient with advanced malignancy.

Manufacturer recommendations and published reports suggest endobronchial valve removal after appropriate air leak resolution, typically after 6 weeks. ${ }^{4}$ Endobronchial valve removal was performed secondary to the known pneumonia risk related to endobronchial valve placement ${ }^{2,3}$ in an attempt to minimize potential complications in this immunocompromised patient. However, after evidence of recurrence and plans for hospice referral, destination therapy appeared quite appropriate. Destination therapy is terminology that has been popularized in end-stage congestive heart failure, utilizing the left ventricular assist device in patients unsuitable for heart transplantation. ${ }^{6}$ Goals of destination therapy include improvement of quality of life, decreased hospitalization, decreased health-care costs, and support of underlying organ failure, all in the context of continued device use until death. ${ }^{7}$ Herein, we reported the first case of endobronchial valve placement as a destination therapy for recurrent pneumothorax in a patient with refractory acute myelogenous leukemia.

\section{REFERENCES}

1. Cerfolio RJ, Tummala RP, Holman WL, Zorn GL, Kirklin JK, McGiffin DC, et al. A prospective algorithm for the management of air leaks after pulmonary resection. Ann Thorac Surg 1998;66(5):1726-1731.

2. Gillespie CT, Sterman DH, Cerfolio RJ, Nader D, Mulligan MS, Mularski RA, et al. Endobronchial valve treatment for prolonged air leaks of the lung: a case series. Ann Thorac Surg 2011;91(1):270-273.

3. Travaline JM, McKenna RJ Jr, De Giacomo T, Venuta F, Hazelrigg SR, Boomer M, et al. Treatment of persistent pulmonary air leaks using endobronchial valves. Chest 2009;136(2):355-360.

4. Mahajan AK, Doeing DC, Hogarth DK. Isolation of persistent air leaks and placement of intrabronchial valves. J Thorac Cardiovasc Surg 2013;145(3):626-630.

5. Baumann MH, Strange C, Heffner JE, Light R, Kirby TJ, Klein J, et al. Management of spontaneous pneumothorax: an American College of Chest Physicians Delphi consensus statement. CHEST 2001; 119(2):590-602.

6. Rose EA, Gelijns AC, Moskowitz AJ, Heitjan DF, Stevenson LW, Dembitsky W, et al. Long-term use of a left ventricular assist device for end-stage heart failure. N Engl J Med 2001;345(20):1435-1443.

7. Yamakawa M, Kyo S, Yamakawa S, Ono M, Kinugawa K, Nishimura T. Destination therapy: the new gold standard treatment for heart failure patients with left ventricular assist devices. Gen Thorac Cardiovasc Surg 2013;61(3):111-117. 